\title{
Photoresponse and morphology of pentacene thin films modified by oxidized and reduced diamond surfaces
}

\author{
W. Gajewski, ${ }^{1}$ M. Huth, ${ }^{2}$ F. Buth, ${ }^{1}$ B. Nickel, ${ }^{2}$ M. Stutzmann, ${ }^{1}$ and J. A. Garrido ${ }^{1}$ \\ ${ }^{1}$ Walter Schottky Institut, Technische Universität München, Garching, Germany \\ ${ }^{2}$ Physics Department and CeNS, Ludwig-Maximilians-Universität, Munich, Germany
}

(Received 17 September 2009; published 9 December 2009)

\begin{abstract}
Because of its large band gap and variety of stable surface terminations, diamond is a suitable material to study the optical and electronic properties of organic films. Optical absorption and photocurrent experiments with pentacene on hydrogen- and oxygen-terminated diamond surfaces reveal a strong, polarization-dependent photoresponse of pentacene films. The diamond surface reconstruction as well as the molecule-surface interactions influence the morphology and the molecular structure of the films, causing the associated polarization dependence. On oxygen-terminated diamond, the pentacene thin-film phase typical for electronically inert substrates such as $\mathrm{SiO}_{2}$ is formed. On hydrogen-terminated diamond, on the other hand, a three-dimensional growth mode of a filamentlike pentacene morphology is observed by atomic force microscopy, with pentacene molecules arranged with their long molecular axis oriented along the hydrogen-terminated diamond surface, as confirmed by x-ray diffraction. Furthermore, on hydrogen-terminated single crystalline diamond, the $\boldsymbol{b}$ axis of the pentacene unit cell is found to orient preferentially perpendicular to the surface, in agreement with photocurrent and optical-absorption experiments.
\end{abstract}

DOI: 10.1103/PhysRevB.80.235311

PACS number(s): 68.55.am, 73.50.Pz, 78.66.Qn

\section{INTRODUCTON}

Pentacene $(\mathrm{Pc})$ has attracted much attention as the prototypical conjugated organic molecule for organic thin-film transistors (OTFTs). Using single crystalline as well as polycrystalline Pc films, carrier mobilities in OTFTs as high as those in amorphous silicon TFTs have been reported. ${ }^{1}$ In order to optimize the transistor characteristics, the structural properties of the polycrystalline Pc films grown by thermal evaporation in ultrahigh vacuum were investigated in detail. ${ }^{1,2}$ In general, two different types of substrates were used: (i) electronically inert substrates such as $\mathrm{SiO}_{2}$ or $\mathrm{Al}_{2} \mathrm{O}_{3}$ and (ii) metals, such as $\mathrm{Au}$ or $\mathrm{Ag}$, and highly oriented pyrolytic graphite (HOPG). The initial growth of pentacene on electronically inert substrates is governed by a twodimensional (2D) (layer-by-layer) growth mode. ${ }^{1}$ In the resulting thin-film phase, the Pc molecules are arranged in an upright orientation, i.e., the long molecular axis points along the surface normal within $7^{\circ} .3$ On the other hand, threedimensional (3D) growth is observed for Pc on metals. ${ }^{4}$ Additionally, several groups have reported a flat-lying first monolayer of $\mathrm{Pc}$ on metal surfaces, attributed to the enhanced interaction between the Pc $\pi$ orbitals and the metal surface. ${ }^{1,5,6}$ Furthermore, by coating the metal surfaces with self-assembled monolayers (SAMs), several groups were able to modify the growth of Pc from $3 \mathrm{D}$ to $2 \mathrm{D} .^{2}$ It has also been confirmed that the observed change from $2 \mathrm{D}$ to $3 \mathrm{D}$ growth induces a change of the pentacene film morphology. ${ }^{2,5,6}$ The understanding of the influence of the growth mode on the optoelectronic properties of $\mathrm{Pc}$ thin films is, however, still poor, mostly due to the variety of different substrates used in previous studies.

In this work, we show that the optoelectronic properties of pentacene thin films can be modulated by controlled modification of diamond surfaces, though the variation of the growth mode of pentacene. In contrast to other semiconduc- tors and metals, both oxygen-terminated and hydrogenterminated diamond surfaces are stable in vacuum and under ambient conditions. While O-terminated diamond surfaces are hydrophilic, H-terminated surfaces are hydrophobic, which allows the preparation of substrates with different surface energies but the same morphology. The surface termination in diamond has a remarkable influence on the surface's electronic properties: while O-terminated surfaces show a positive electron affinity, H-terminated surfaces exhibit a negative electron affinity. ${ }^{7}$ Thus, the surface termination can also be used to tune the position of the diamond conduction and valence bands at the surface. This has been applied to induce charge transfer between the diamond surface and the highest occupied molecular orbital (HOMO) or lowest unoccupied molecular orbital (LUMO) levels of organic molecules such as fullerenes. ${ }^{8}$ Additionally, due to the large band gap of diamond (5.45 eV at room temperature), the optical and optoelectronic characterization of the deposited organic films is not limited by the substrate. Therefore, diamond surfaces are ideal substrates to investigate optoelectronic properties of organic thin films, since they combine the properties of standard electronically inert surfaces such as $\mathrm{SiO}_{2}$ for oxidized diamond surfaces and, on the other hand, electronically active surfaces, such as graphite or metals, when the surface is hydrogenated.

\section{EXPERIMENTAL}

Commercially available intrinsic (100)- and (111)oriented single-crystal diamond (SCD) and polycrystalline diamond (PCD) films were chemically oxidized in a boiling $\mathrm{H}_{2} \mathrm{SO}_{4}+\mathrm{KNO}_{3}$ solution for $1 \mathrm{~h}$ or hydrogenated by exposure to atomic hydrogen generated by a hot wire in a highvacuum chamber at a substrate temperature of about $550{ }^{\circ} \mathrm{C}$. The diamond films have a very smooth surface, with a typical roughness (rms) of 0.3 and $0.6 \mathrm{~nm}$ for SCD and PCD, 


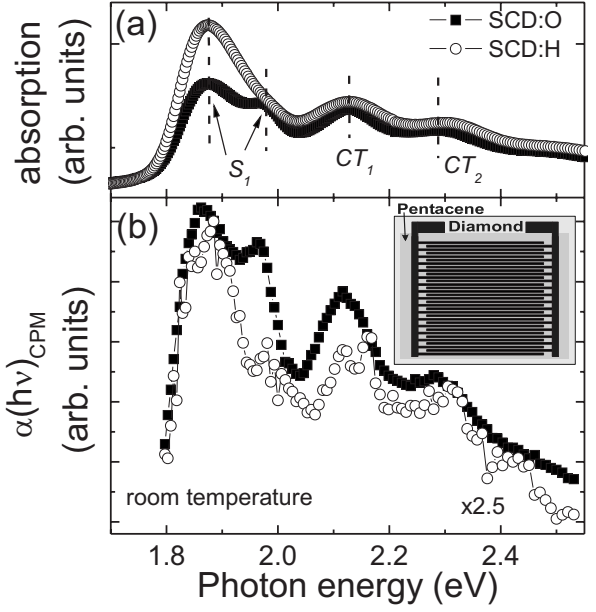

FIG. 1. (a) Room temperature optical absorption and (b) CPM spectra of Pc films on SCD:O and SCD:H. For better comparison, the CPM spectrum of Pc on SCD:H is multiplied by a factor of 2.5. Inset of (b) shows a top view on the diamond-pentacene sample. The absorption maxima are attributed to singlet exciton $\left(S_{1}\right)$ Davydov doublet (1.84 and $1.96 \mathrm{eV}$ ) and CT excitons (2.11 and $2.27 \mathrm{eV})$.

respectively. For optoelectronic characterization, Ti/Au (20/ $200 \mathrm{~nm}$ ) interdigitated metal contacts (spacing of $45 \mu \mathrm{m}$ ) were evaporated on the substrates. Prior to pentacene deposition (rate $0.05-0.1 \AA / \mathrm{s}$ ), the diamond films were annealed in situ at $480 \mathrm{~K}$ (base pressure of $10^{-7} \mathrm{mbar}$ ). Pentacene films with a nominal thickness of $50 \mathrm{~nm}$ (measured using a quartz microbalance) were deposited simultaneously on $\mathrm{H}$ and $\mathrm{O}$-terminated diamond substrates. $\mathrm{SiO}_{2}$ substrates were used for calibration and reference. The optical-absorption measurements between 2000 and $200 \mathrm{~nm}$ were done under ambient conditions using a Perkin Elmer $\lambda-900$ spectrometer. Constant photocurrent spectroscopy (CPM) (Ref. 9) was used to investigate the optoelectronic properties of the pentacene films. The CPM measurements were performed in vacuum $\left(10^{-6}\right.$ mbar) using chopped $(4 \mathrm{~Hz})$ monochromatic light from a xenon arc lamp for optical excitation in the energy range $1.8-2.8 \mathrm{eV}$. In contrast to conventional photocurrent spectroscopy, CPM probes the spectral variation of the absorption coefficient; this is done by adjusting the light intensity so that the photocurrent is kept constant at different wavelengths. The prepared diamond/pentacene samples were illuminated over the whole device structure (about 2.3 $\times 2.3 \mathrm{~mm}^{2}$ ) from the pentacene layer side. The morphology of the Pc films was investigated with AFM in tapping mode under ambient conditions and by $\mathrm{x}$-ray diffraction experiments, which were performed at the synchrotron $\mathrm{x}$-ray facility HASYLAB (Hamburg, Germany).

\section{RESULTS AND DISCUSSION}

Typical absorption spectra of 50-nm-thick Pc films deposited on oxygen- (SCD:O) and hydrogen-terminated (SCD:H) surfaces of single crystalline diamond are depicted in Fig. 1(a). In the case of the Pc films on SCD:O substrates, the absorption spectra is similar to those reported previously for single crystalline pentacene ${ }^{10}$ or polycrystalline thin films prepared on inert surfaces, ${ }^{11-13}$ revealing the Davydov doublet due to Pc singlet exciton absorption $\left(S_{1}\right)$ at 1.84 and 1.96 $\mathrm{eV}$ as well as the charge-transfer (CT) excitons ${ }^{14}$ at 2.11 and $2.27 \mathrm{eV}$. In the case of Pc films on SCD:H, however, the $S_{1}$ absorption peak with it maximum at $1.85 \mathrm{eV}$ is broader and the peak at $1.96 \mathrm{eV}$ has almost vanished. Also, the peak assigned to charge-transfer exciton is broader and is slightly shifted to $2.12 \mathrm{eV}$, as compared to Pc films on SCD:O. The constant photocurrent method (CPM) was applied to investigate whether the observed differences in the absorption spectra of the pentacene films on $\mathrm{O}$ - and $\mathrm{H}$-terminated diamond surfaces are also reflected in the optoelectronic properties of the investigated diamond/pentacene heterostructures. ${ }^{9}$ The photoresponse of the films can be directly related to the absorption features observed in Fig. 1(a) for both diamond surface terminations, indicating that all excitations, including the excitonic peaks, are electronically active. The CPM spectra of pentacene films on SCD:O and SCD:H [Fig. 1(b)] closely follow the corresponding absorption spectra [Fig. 1(a)]. Note, however, that the CPM signal for the pentacene films on SCD:H surfaces is lower by a factor of 2.5. This can be attributed to the higher dark current of the $\mathrm{H}$-terminated diamond substrate due to the surface conductivity of hydrogenated diamond. ${ }^{7}$ The higher background current results in a decrease of the photocurrent sensitivity, which may partly contribute to the lowering of the CPM signal. For Pc films on SCD:O, the CPM signal maxima at 1.87 and $1.98 \mathrm{eV}$ correspond to the Davydov doublet of the $S_{1}$ exciton observed in the absorption spectra. In addition, the positions of the charge-transfer excitons are in good agreement with those estimated from the absorption spectra. For Pc films on $\mathrm{SCD}: \mathrm{H}$, a broad absorption peak is observed in the CPM spectrum [Fig. 1(b)] at about $1.86 \mathrm{eV}$ and, as in the case of the absorption spectra [Fig. 1(a)], the presence of the second peak of the Davydov doublet at about $1.96 \mathrm{eV}$ is either weak, absent, or in the shoulder of the peak at lower energy. The polarization dependence of the light absorption observed in other aromatic molecules such as anthracene shows the highest absorption when the electric field of the incoming light is parallel to one of the short crystal axes $\boldsymbol{a}$ and $\boldsymbol{b} .{ }^{10}$ The polarization-dependent absorption of pentacene films has recently been observed by ellipsometry experiments using single crystalline pentacene crystals. ${ }^{15}$ Accordingly, the presence of the Davydov $S_{1}$ doublet as observed here for Pc on oxidized diamond suggest that the $\boldsymbol{a}-\boldsymbol{b}$ plane is parallel to the polarization plane of the incident light and thereby parallel to the diamond surface. This result is in agreement with the broadly accepted model of the pentacene thin-film growth on electronically inert surfaces such as $\mathrm{SiO}_{2}$, where typically an upright standing pentacene phase has been observed. ${ }^{2}$ The decrease of the Davydov doublet intensity for pentacene films on the H-terminated diamond films reported here suggests a different molecular arrangement, i.e., no upright orientation of the long molecular axis and in particular, the $\boldsymbol{b}$-axis oriented perpendicular to the surface.

In order to verify the conclusions made above and to correlate the observed optical-absorption and photocurrent data with the Pc films structure, we have performed a detailed study of the pentacene film morphology. Typical AFM micrographs of Pc film morphology on oxidized and hydrogen- 


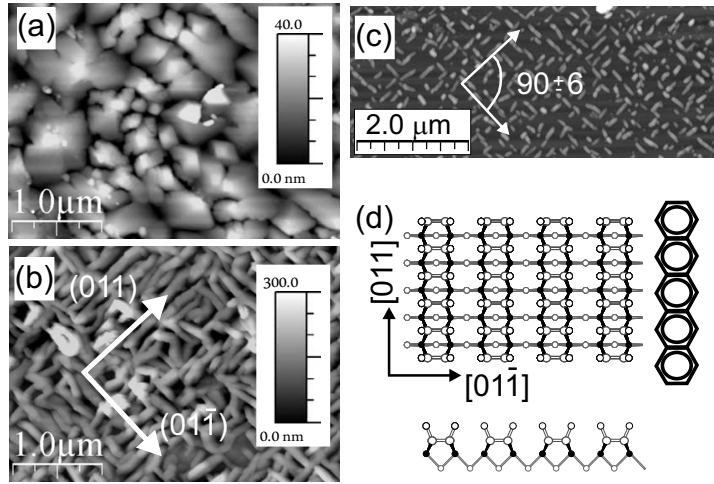

FIG. 2. AFM micrographs of pentacene films on the (a) oxidized and [(b) and (c)] hydrogenated surfaces of SCD. Nominal film thicknesses are [(a) and (b)] $50 \mathrm{~nm}$ and (c) $0.8 \mathrm{~nm}$. On the oxidized surface, pentacene grains with sizes up to $1 \mu \mathrm{m}$ are observed, exhibiting a terraced structure with a step spacing of about $1.5 \mathrm{~nm}$. A filamentlike structure (average height: $150 \mathrm{~nm}$ ) is observed for the $\mathrm{H}$-terminated diamond. The main crystallographic directions parallel to the SCD:H surface are indicated by arrows. (d) shows a schematic top and side view of the $(2 \times 1)$ reconstruction of the $(100)$ diamond surface. A pentacene molecule is shown for comparison.

ated surfaces of single crystalline diamond films are presented in Fig. 2. The Pc texture observed on oxidized diamond [Fig. 2(a)] is very similar to the one on inert substrates such as $\mathrm{SiO}_{2} \cdot{ }^{1-3,16}$ For films with a nominal thickness of $50 \mathrm{~nm}$, the formation of terraced pyramidal structures (grain size $\sim 0.5-1 \mu \mathrm{m}$ ) is seen. The step heights observed by AFM are in good agreement with the $d_{(00 l)}$ distance of the thin-film phase of pentacene. ${ }^{2}$ The morphology of the pentacene film on the hydrogen-terminated diamond surface [Fig. 2(b)] is entirely different. Filamentlike islands typical for a $3 \mathrm{D}$ growth mode are observed. The average height of the Pc structures is about $150 \mathrm{~nm}$, with a length of $0.4-0.5 \mu \mathrm{m}$. Furthermore, two preferential orientations are observed on the SCD:H surface with an angle of $90^{\circ}$ relative to each other. For the (100)-oriented SCD $[\operatorname{SCD}(100)]$ used for this study, the main crystallographic directions parallel to the surface are the (011) and (01-1), marked with arrows in Fig. 2(b). For the H-terminated diamond surface SCD(100)-(2 $\times 1): \mathrm{H}$, these directions correspond to the directions of the dimer rows of the $(2 \times 1)$ reconstructed surface [Fig. $2(\mathrm{~d})] .{ }^{17}$ In addition, they are in good agreement with the preferential direction of the filamentlike texture of the pentacene islands observed in the atomic force microscopy (AFM) micrographs. A similar filamentlike morphology has been observed for Pc films on single crystalline and polycrystalline $\mathrm{Au}(111){ }^{4,5}$ Recently, Koini et al. reported an influence of the surface corrugation of weakly interacting oxygen-passivated $\mathrm{Cu}(100)$ single crystals on the morphology pattern of pentacene films. ${ }^{18}$ These authors observed preferential alignment of the pentacene molecules with the long crystal axis, $\boldsymbol{c}$, along the surface corrugation due to the presence of the oxygen atoms. In analogy, the filamentlike island morphology of the Pc films on SCD (100)- $(2 \times 1): \mathrm{H}$ surface can be assigned to the influence of surface corrugation of the H-terminated diamond due to the presence of the dimer rows. In order to verify this hypothesis, additional growth experiments have
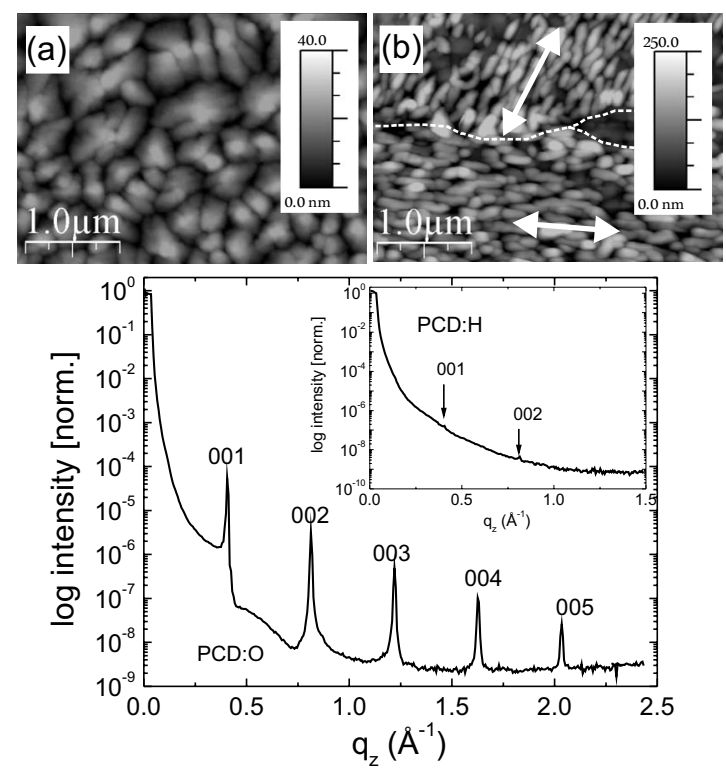

FIG. 3. AFM micrographs of pentacene films grown on the (a) oxidized and (b) hydrogenated surfaces of PCD used for the x-ray experiments. (c) Out-of-plane x-ray reflectivity pattern of pentacene on PCD:O. Inset shows the out-of-plane x-ray reflectivity pattern of pentacene on PCD:H.

been performed on the H-terminated (100)- and (111)oriented surfaces of SCD films, $\operatorname{SCD}(100)-(2 \times 1): \mathrm{H}$ and $\mathrm{SCD}(111)-(1 \times 1): \mathrm{H}$, respectively. Deposition of a $0.8 \mathrm{~nm}$ thin pentacene film allowed us a detailed investigation of the orientation and shape of the pentacene structures. For the $\operatorname{SCD}(100)-(2 \times 1): \mathrm{H}$ substrate [Fig. 2(c)], the filamentlike structures are again perpendicular to each other, in agreement with the thicker films [Fig. 2(b)]. The main symmetry directions of the $\operatorname{SCD}(100)-(2 \times 1): H$ surface have been marked with arrows in the schematic model representing the top and side views of the diamond surface in Fig. 2(d). In the case of the (111) diamond surface, with a typical $(1 \times 1)$ reconstruction, ${ }^{7}$ filamentlike growth of the pentacene layer in three rotational domains with a rotation angle close to $60^{\circ}$ is observed (data not shown), as expected from the symmetry of this surface reconstruction. Thus, our data confirm a strong influence of the diamond surface symmetry on the directional growth of the pentacene thin film on H-terminated diamond.

For a verification of the pentacene molecular arrangement induced by the different diamond surface terminations, observed by the AFM experiments, x-ray diffraction experiments have been conducted. The initial experiments were performed on 50-nm-thick pentacene films deposited on polycrystalline diamond substrates $(\mathrm{PCD}, \mathrm{O}-$ and H-terminated). As depicted in Figs. 3(a) and 3(b), the morphology of pentacene films on oxygen- and hydrogenterminated PCDs is similar to Pc on SCD surfaces [Fig. 2]. A filamentlike structure and preferential orientation of the pentacene filaments is observed for PCD:H substrates, where the presence of the grain boundaries [marked in dashed line in Fig. 3(b)] appears as an orientation-domain boundary. A Debye-Scherrer image of a PCD:H substrate depicted as the inset of [Fig. 4(a)] shows powderlike rings, as expected for a 

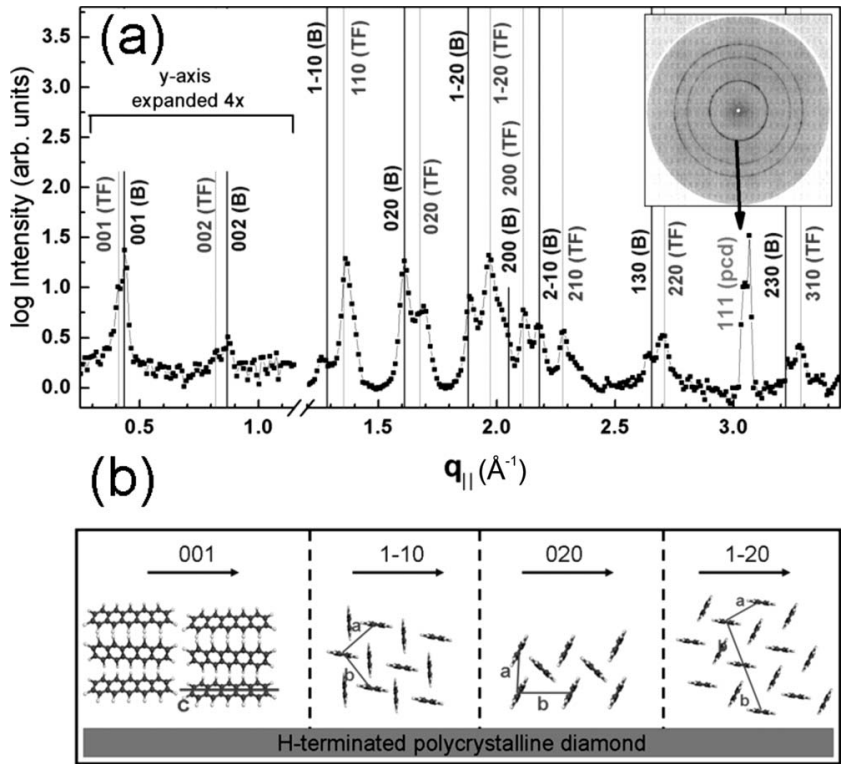

FIG. 4. (a) In-plane XRD data for Pc film on PCD:H. Vertical lines mark the calculated positions of the main diffraction peaks of the bulk phase (b) and the TF phase. In the low $q_{\|}$range, the vertical scale has been expanded 4 times for clarity. A Debye-Scherrer image of a PCD:H substrate (inset) shows powder rings. (b) shows schematic side views of selected orientations of pentacene on hydrogenated diamond. Cross sections along 001, 1-10, 020, and 1-20 directions are depicted. For all schematics, the orientations of the $\boldsymbol{a}, \boldsymbol{b}$, and/or $\boldsymbol{c}$ axes have been shown for clarity.

polycrystalline material without any preferential orientation. The results of the x-ray reflectometry experiments for both PCD surface terminations are shown in Fig. 3(c). For the oxidized diamond surfaces [inset in Fig. 3(c)], the graph reveals a clear Bragg pattern with peak separations corresponding to a layer spacing of $d_{(00 l)}=1.54 \pm 0.01 \mathrm{~nm}$. From the evaluation of the full width at half maximum $\left(0.019 \pm 0.002 \AA^{-1}\right)$ of the XRD spectra, a crystalline grain size along the surface normal of about $33 \mathrm{~nm}$ is deduced for Pc on PCD:O. Thus, the presence of the upright standing pentacene phase typical for electronically inert surfaces ${ }^{3,16}$ is confirmed for the O-terminated diamond substrates. This is consistent with the data obtained from the optoelectronic characterization, supporting a parallel orientation of the crystal $\boldsymbol{a}-\boldsymbol{b}$ plane with respect to the diamond surface. The x-ray out-of-plane reflectivity spectrum of Pc on PCD:H reveals only very weak $(00 l)$ Bragg peaks at the position for the upright standing Pc molecules. The presence of a very small fraction of the standing phase on SCD:H and PCD:H surfaces is assigned to a nonperfect hydrogenation process, which can result in the presence of a few oxygen-terminated sectors in nominally hydrogenated surfaces. Complementary in-plane XRD diffraction, depicted in Fig. 4(a), has been used to further characterize the Pc ordering for the hydrogenated diamond surface. (001) reflections occur in the in-plane XRD pattern, which indicates that the long molecular axis $(\boldsymbol{c}$ axis) is parallel to the sample surface. This also has been observed for Pc on HOPG ${ }^{20}$ gold, ${ }^{5}$ and oxygen-passivated copper surfaces. ${ }^{18}$ Furthermore, the (001) reflex is split, which suggests the presence of different pentacene poly-

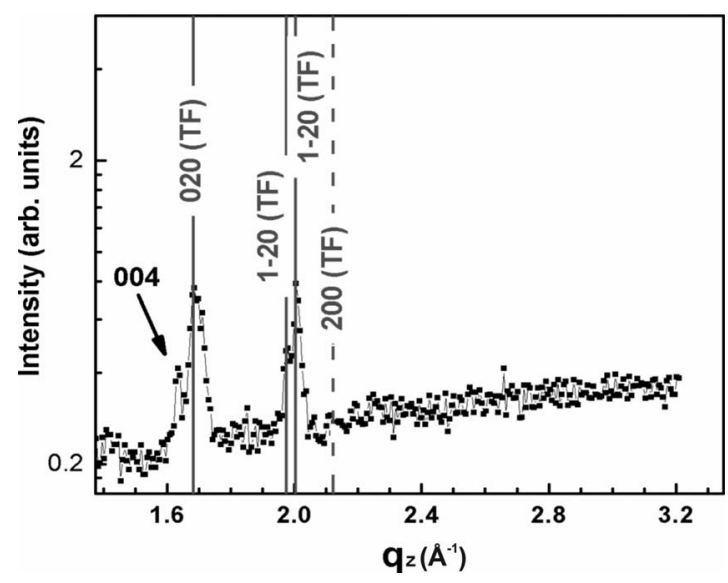

FIG. 5. Zoom into the out-of-plane x-ray reflectivity pattern of pentacene on SCD:H. Note that the (020) and the (120), (1-20) planes show Bragg reflections, whereas the (200) peak is not observed, indicating the $\boldsymbol{a}$ axis being mainly parallel and the $\boldsymbol{b}$ axis being mainly perpendicular to the surface.

morphs. The $d_{00 l}$ spacing $\left(d=2 \pi / q: d_{00 l}=15.4\right.$ and $14.5 \AA$, respectively) indicates the presence of a bulk phase and a thin-film phase polymorph. Using the structure of these polymorphs reported in the literature,, 319 all Bragg peaks can be attributed to a specific polymorph, either thin-film phase $(\mathrm{TF})$ or bulk phase $(B)$, and indexed. In order to refine the structural information, the measured peak positions were fitted to simulated peak values using a combination of a Monte Carlo method and a Levenberg-Marquardt nonlinear leastsquares fit routine. ${ }^{21}$ The best fits obtained for the unit-cell parameters of the thin-film structure are $a=5.96 \AA, b$ $=7.60 \AA, c=15.33 \AA, \alpha=80.88^{\circ}, \beta=87.18^{\circ}$, and $\gamma=90.1^{\circ}$, whereas the bulk phase structure parameters are $a=6.29 \AA$, $b=7.82 \AA, \quad c=14.50 \AA, \quad \alpha=86.94^{\circ}, \quad \beta=76.93^{\circ}, \quad$ and $\gamma$ $=91.15^{\circ}$. These values are in good agreement with the values for the bulk pentacene polymorph, reported by Campbell et $a l .{ }^{19}$ and the values for the thin-film pentacene polymorph, reported by Schiefer $e t$ al.,${ }^{3}$ respectively. A sketch of selected orientations of Pc molecules based on the observed peaks is depicted in Fig. 4(b). The arrangement of the pentacene molecules partly explains the differences observed in the opticalabsorption and photocurrent spectra of Pc films deposited on $\mathrm{O}$ - and H-terminated diamond surfaces. The absorption and photocurrent spectra of the pentacene film on SCD:H surfaces show a strong intensity decrease of the higher-energy peak of $S_{1}$ at $1.96 \mathrm{eV}$. An almost perpendicular arrangement of the $\boldsymbol{b}$ axis would explain the absence of the $1.96 \mathrm{eV}$ peak in the absorption spectra. ${ }^{10,15}$ The in-plane x-ray data on PCD:H in the available $q_{z}$ range do not reveal a clear preference for an orientation of the $\boldsymbol{a}$ or $\boldsymbol{b}$ axis. However, the observed lack of the Bragg series characteristic for an upright standing pentacene film does not unequivocally rule out an arrangement with the $c$ axis of the Pc crystal aligned parallel to the H-terminated diamond surface.

To confirm this experimentally, out-of-plane x-ray reflectivity measurements have been performed on a 50-nm-thick pentacene film grown on (100) SCD:H (Fig. 5) in the extended $q_{z}$ range. The indexed peaks correspond to the (020), the (120), and the (1-20) crystal planes of pentacene. At the 
$q$ position of the (200) plane, no peak is observed, which suggests that the $\boldsymbol{a}$ axis is mainly parallel to the surface, while the $\boldsymbol{b}$ axis is mainly perpendicular to the surface. This is in good agreement with the optical measurements performed on Pc films on SCD:H substrates and also confirms our growth model depicted schematically in Fig. 4(b).

The nature of the molecule-surface interactions governing the different molecular structure of the Pc films is discussed in the following. First of all, the different values of the surface free energy of pentacene and diamond surfaces with different termination should be considered as a possible explanation of the observed differences in the pentacene film morphology. The growth orientation of pentacene thin films results from the competition between Pc crystal planes with different surface energies. As suggested by Northrup et al., ${ }^{16}$ the lowest surface energy corresponds to the (001) plane of a pentacene crystal and is 1.5 times lower than that of (100) or (110) planes. Therefore, on electronically inert surfaces such as O-terminated diamond, pentacene growth shows a (001) layer-by-layer structure. Yoshikawa et al. ${ }^{22}$ modified the surface energy by using self-assembled monolayers on $\mathrm{SiO}_{2}$ surfaces. In the case of low surface energies, the authors observed a spontaneous aggregation of pentacene films which led to the formation of long filamentlike structures next to bare $\mathrm{SiO}_{2}$ substrate regions. In the case of hydrogenated diamond, the surface free energy is relatively low compared to oxidized diamond surfaces ${ }^{23}$ or the $\mathrm{SiO}_{2}$ surface. This difference in the surface energy could explain the observed filamentlike structures on H-terminated diamond surfaces. ${ }^{22}$ Second, the formation of a chemical bond between the pentacene $\pi$ orbitals and a surface dangling bond could be considered as the origin of the preferential nucleation, similar to the $(2 \times 1)$ reconstructed $\mathrm{Si}$ surface. ${ }^{24}$ The low density of dangling bonds on the H-terminated diamond surface, though, makes chemisorption induced by dangling bonds not very probable. However, a strong electronic interaction between the Pc molecules and the substrate surface could also explain the preferential orientation observed for H-terminated diamond surfaces. Such strong electronic interactions have been reported between metallic surfaces and various molecular $\pi$ systems, including pentacene. ${ }^{4}$ Thus, we tentatively assign the corrugation of the electronic structure of the hydrogenated (100) diamond surface, which results from the dimer reconstruction, to be responsible for the preferential orientation of the filamentlike structures. The alignment of the long molecular axis of pentacene on the diamond surface along the dimer rows was confirmed in Fig. 2, where the angle measured for the pentacene filaments directions is in close agreement with the directions of the dimer rows of the $\operatorname{SCD}(100)-(2 \times 1): H$ surface. Such a specific alignment of pentacene molecules seems to be consistent with the accepted model of the formation of molecular crystals, ${ }^{10}$ where dispersive forces, dipole-dipole interaction, and hydrogen bonds formation govern the crystal growth.

\section{SUMMARY}

In summary, we have shown that diamond surfaces are excellent platforms to manipulate the morphology and the optoelectronic properties of pentacene thin films. Our experiments demonstrate that the surface termination of diamond determines the growth mode of pentacene: quasi-2D growth on O-terminated surfaces and 3D growth on H-terminated surfaces. The observed change of the Pc film morphology is considered to be responsible for the differences in the optoelectronic properties of the pentacene films and manifested in a polarization-dependent optical absorption of the pentacene films. For O-terminated diamond, the presence of an upright standing thin-film phase has been observed, with a characteristic interlayer spacing, $d_{(00 l)}$, of $1.54 \pm 0.01 \mathrm{~nm}$ and a strong Davydov doublet of the singlet exciton absorption. Both diamond surface terminations result in different surface energies, but also different electronic properties due to the influence of $\mathrm{C}-\mathrm{H}$ and $\mathrm{C}-\mathrm{O}$ interfacial dipoles. By changing the surface termination to hydrogen, the growth of filamentlike structures of pentacene in a bulklike crystal phase has been induced, characterized by a suppressed Davydov doublet component. Complementary x-ray experiments have confirmed the presence of the dominant lying-down phase on the hydrogen-terminated diamond films with the $c$ axis oriented parallel to the diamond surface. Furthermore, for $\operatorname{SCD}(100)-(2 \times 1): H$ substrates, a preferential orientation of the pentacene $\boldsymbol{a}$ axis parallel and $\boldsymbol{b}$ axis perpendicular to the diamond surface has been found, explaining the differences of the optical properties of pentacene on both surface terminations.

\section{ACKNOWLEDGMENTS}

This work was supported by the EC Research Training Network "DRIVE" under Contract No. RTN-CT-2004512224 and by the EU-STREP Contract No. 032652 BIODOT. The "Nanosystems Initiative Munich" (NIM) is gratefully acknowledged.

\footnotetext{
${ }^{1}$ R. Ruiz, B. Nickel, N. Koch, L. C. Feldman, R. F. Haglund, A. Kahn, and G. Scoles, Phys. Rev. B 67, 125406 (2003).

${ }^{2}$ R. Ruiz, D. Choudhary, B. Nickel, T. Toccoli, K.-C. Chang, A. C. Mayer, P. Clancy, J. M. Blakely, R. L. Headrick, S. Iannotta, and G. G. Malliaras, Chem. Mater. 16, 4497 (2004).

${ }^{3}$ S. Schiefer, M. Huth, A. Dobrinevski, and B. Nickel, J. Am. Chem. Soc. 129, 10316 (2007).
}

${ }^{4}$ D. Käfer, L. Ruppel, and G. Witte, Phys. Rev. B 75, 085309 (2007).

${ }^{5}$ D. Käfer and G. Witte, Chem. Phys. Lett. 442, 376 (2007).

${ }^{6}$ L. Casalis, M. F. Danisman, B. Nickel, G. Bracco, T. Toccoli, S. Iannotta, and G. Scoles, Phys. Rev. Lett. 90, 206101 (2003).

${ }^{7}$ J. Ristein, Appl. Phys. A: Mater. Sci. Process. 82, 377 (2006).

${ }^{8}$ P. Strobel, M. Riedel, J. Ristein, and L. Ley, Nature (London) 
430, 439 (2004).

${ }^{9}$ M. Vanecek, J. Kocka, J. Stuchlik, and A. Triska, Solid State Commun. 39, 1199 (1981).

${ }^{10}$ M. Pope and C. E. Swenberg, Electronic Processes in Organic Crystals and Polymers, 2nd ed. (Oxford University Press, New York, 1999), p. 1328, ISBN 0195129636.

${ }^{11}$ D. Knipp, D. K. Murti, B. Krusor, R. Apte, L. Jiang, J. P. Lu, B. S. Ong, and R. A. Street, Mater. Res. Soc. Symp. Proc. 665, C5.44.1 (2001).

${ }^{12}$ M. Breban, D. B. Romero, S. Mezhenny, V. W. Ballarotto, and E. D. Williams, Appl. Phys. Lett. 87, 203503 (2005).

${ }^{13}$ J. Gao and F. A. Hegmann, Appl. Phys. Lett. 93, 223306 (2008).

${ }^{14}$ L. Sebastian and G. Weiser, Chem. Phys. 61, 125 (1981).

${ }^{15}$ M. Dressel, B. Gompf, D. Faltermeier, A. K. Tripathi, J. Pflaum, and M. Schubert, Opt. Express 16, 19770 (2008).

${ }^{16}$ J. E. Northrup, M. L. Tiago, and S. G. Louie, Phys. Rev. B 66,
121404(R) (2002).

${ }^{17}$ J. Ristein, Surf. Sci. 600, 3677 (2006).

${ }^{18}$ M. Koini, T. Haber, O. Werzer, S. Berkebile, G. Koller, M. Oehzelt, M. G. Ramsey, and R. Resel, Thin Solid Films 517, 483 (2008).

${ }^{19}$ R. B. Campbell, J. M. Robertson, and J. Trotter, Acta Crystallogr. 14, 705 (1961).

${ }^{20}$ N. Koch, A. Vollmer, I. Salzmann, B. Nickel, H. Weiss, and J. P. Rabe, Phys. Rev. Lett. 96, 156803 (2006).

${ }^{21}$ D. W. Marquardt, J. Soc. Ind. Appl. Math. 11, 431 (1963).

${ }^{22}$ G. Yoshikawa, J. T. Sadowski, A. Al-Mahboob, Y. Tsuruma, S. Ikeda, and K. Saiki, Appl. Phys. Lett. 90, 251906 (2007).

${ }^{23}$ L. Ostrovskaya, V. Perevertailo, V. Ralchenko, A. Dementjev, and O. Loginova, Diamond Relat. Mater. 11, 845 (2002).

${ }^{24}$ D. Choudhary, P. Clancy, and D. R. Bowler, Surf. Sci. 578, 20 (2005). 